\title{
Multiple mechanisms mediate cholesterol-induced synaptogenesis in a CNS neuron
}

\author{
Christian Goritz, Daniela H. Mauch, and Frank W. Pfrieger* \\ Max-Planck/CNRS Group, UPR 2356, Centre de Neurochimie, 5, rue Blaise Pascal, F-67084 Strasbourg, France
}

Received 1 December 2004; revised 9 February 2005; accepted 9 February 2005

Available online 1 April 2005

\begin{abstract}
Neurons undergo a complex differentiation process that endows them with the ability to generate electrical signals and to transmit them via synaptic connections. There is increasing evidence that glial cells regulate specific aspects of neuronal differentiation including synapse formation, but the underlying mechanisms are not well understood. Here, we show how glia-derived cholesterol promotes the development of synapses in microcultures of highly purified retinal ganglion cells (RGCs) from postnatal rats. We identify dendrite differentiation as rate limiting step for glia-induced synaptogenesis and we show that this process requires cholesterol. Furthermore, we show that cholesterol enhances directly presynaptic differentiation and that it is essential for continuous synaptogenesis and for the stability of evoked transmitter release. These results reveal new roles of cholesterol in neuronal differentiation and underline the importance of neuron-glia interactions during brain development.

(C) 2005 Elsevier Inc. All rights reserved.
\end{abstract}

\section{Introduction}

Glial cells control different aspects of brain development (Campbell and Gotz, 2002; Gomes et al., 2001; Lemke, 2001; Nadarajah and Parnavelas, 2002), but the formation of synapses, one of the last steps in neuronal differentiation, has long been thought to involve neurons only. Increasing evidence that glial cells influence synapse development (Koirala et al., 2003; Slezak and Pfrieger, 2003; Ullian et al., 2004a) prompts a revision of this view (Goda and Davis, 2003; Li and Sheng, 2003; Sanes and Lichtman, 1999; Ziv and Garner, 2004). In vitro studies have shown that soluble and contact-dependent signals from astrocytes stimulate the formation of synapses in neurons from hippocampus (Hama et al., 2004), retina (Mauch et al., 2001; Nagler et al., 2001; Ullian et al., 2001) and spinal cord (Peng et al., 2003; Ullian et al., 2004b) without affecting neuronal survival. Furthermore, there is evidence that glial cells promote the maturation of synaptic connections

\footnotetext{
* Corresponding author. Fax: +33 388601664 .

E-mail address: fw-pfrieger@gmx.de (F.W. Pfrieger).

Available online on ScienceDirect (www.sciencedirect.com).
}

(Mazzanti and Haydon, 2003; Nagler et al., 2001; Ullian et al., 2001) and support their structural and functional stability (Reddy et al., 2003; Slezak and Pfrieger, 2003; Ullian et al., 2001).

Studies on RGCs, which can be immunoisolated from postnatal rats (Barres et al., 1988) and cultured under defined conditions (Meyer-Franke et al., 1995), revealed that signals from macroglial cells strongly raise the level of synaptic activity (Pfrieger and Barres, 1997) by promoting the formation and maturation of synapses in an activity-independent manner (Nagler et al., 2001; Ullian et al., 2001). Subsequently, our group identified cholesterol as a glia-derived factor that mediates synapse-promoting effects (Mauch et al., 2001). Cholesterol is secreted by astrocytes via apolipoprotein E-containing lipoproteins (Pfrieger, 2003a). So far, however, it remained unclear how cholesterol enhances the formation and function of synapses. Here, we show that dendrite differentiation is the rate-limiting step for glia-induced synaptogenesis in RGCs and that this process requires cholesterol. In addition, we show that cholesterol enhances directly presynaptic differentiation, supports continuous synapse development and is essential for the stability of evoked synaptic transmission.

\section{Results}

We studied how cholesterol influences synapse development in microcultures of highly purified rat RGCs growing singly or in small groups of up to 12 cells (Mauch et al., 2001; Nagler et al., 2001). To determine whether cholesterol alone mimicked the effects of soluble glial factors on synapses, we treated microcultures of RGCs in parallel with glia-conditioned medium (GCM) or with cholesterol at the same concentration $\left(5 \mu \mathrm{g} \mathrm{ml}^{-1}\right)$ at which it is present in GCM (Mauch et al., 2001).

Time course of GCM- and cholesterol-induced effects on synapses

As a first step, we analyzed how fast GCM and cholesterol induce the different effects on synapses that we reported previously (Mauch et al., 2001). We added GCM or cholesterol to 5 day old microcultures of RGCs and examined after different periods of treatment the number and activity of synapses in neurons growing 
in microcultures. Control cells were cultured for the entire period in defined culture medium. To determine the level of synaptic activity, we recorded spontaneous, asynchronous and evoked excitatory postsynaptic currents (EPSCs) (Nagler et al., 2001). As shown in Fig. 1, GCM and cholesterol raised the frequencies of spontaneous and asynchronous release and the size of miniature and evoked EPSCs to the same extent and with a similarly slow time course. GCM and cholesterol induced large miniature EPSCs with charge transfers exceeding $100 \mathrm{fC}$ (Fig. 1C), but these occurred only in about $50 \%$ of the neurons tested (Chol: 15 out of 28; GCM: 10 out of 27). Since frequencies and charge transfer amplitudes are asymmetrically distributed, boxplots were chosen to represent data. Calculated mean frequencies under glia-free conditions and after long-term treatment with GCM or cholesterol are similar to those reported previously (Mauch et al., 2001; Nagler et al., 2001).

We next determined whether cholesterol mediated the GCMinduced increase in release efficacy that we reported previously (Nagler et al., 2001). To accomplish this, we tested its effect on synaptic responses to paired-pulse stimulation, which are sensitive to changes in the efficacy of transmitter release (Zucker and Regehr, 2002). Under control conditions, pairedpulse ratios (PPRs) averaged around 0.6 regardless of the interstimulus interval (ISI). Treatment of RGCs with cholesterol or GCM induced robust ISI-dependent paired-pulse depression (PPD) (Fig. 2). In contrast to other synaptic changes, PPD occurred already after 1 day of treatment indicating a fast change in the release efficacy. This confirmed our previous observation that GCM decreases the failure rate within $24 \mathrm{~h}$ (Nagler et al., 2001) and indicated that cholesterol contained in GCM induced this effect.

Next, we resolved how fast GCM and cholesterol enhanced the number of synapses. We double-stained synapses with antibodies against synapsin I and glutamate receptor $2 / 3$ (GluR2/3) after different periods of treatment with GCM or cholesterol and determined the density of immunostained puncta in neurites by an automatic routine. As shown in Fig. 3, GCM and cholesterol enhanced the number of synapsin-, GluR2/3positive and of double-stained puncta per neurite area with remarkably similar time courses. As for the effects on synaptic activity shown in Fig. 1, statistically significant changes required at least 3 days of treatment. However, we noted two differences

Fig. 1. GCM- and cholesterol-induced enhancement of synaptic activity requires at least 3 days of treatment. Boxplots showing the frequencies of spontaneous (A) and asynchronous EPSCs (B) in RGCs cultured for 5 days in defined medium and then for 1,2 or 3 to $7(3+)$ days in defined medium (RGC; 60 neurons with activity out of 97 tested), in the presence of GCM (1: 18 of $28 ; 2: 19$ of $34 ; 3+: 101$ of 124) or in the presence of cholesterol (Chol; 1: 14 of $30 ; 2: 25$ of 35; 3+: 112 of 136). In boxplots, horizontal lines represent the median, lower and upper box limits show 1st and 3rd quartile, respectively and whiskers indicate the 1.5 -fold interquartile range. (C) Charge transfer amplitudes of miniature EPSCs (quantal size) in RGCs cultured as described above in defined medium (RGC; 7 neurons $/ 28$ events), in GCM (1: 5/23; 2: 7/105; 3+: 28/901) or in cholesterol (Chol; 1 : $5 / 33 ; 2: 7 / 909 ; 3+: 29 / 2127)$. Filled circles indicate values outside the 1.5 fold interquartile range. (D) Charge transfer of evoked EPSCs in RGCs cultured as described above in defined medium (RGC; $n=14$ neurons), in GCM (1: 10;2: 9; 3+: 71) or in cholesterol (Chol; 1: 7; 2: 14;3+: 67). Asterisks indicate significant changes compared to control cultures (see Experimental methods; (A, B) Kruskal-Wallis test; (C, D) ANOVA, Dunnett's post hoc test). between cholesterol- and GCM-treated cells: first, cholesterol induced a smaller, but still significant increase in synapse number than GCM and second, in cholesterol-treated cultures, synapsinpositive puncta appeared dispersed in neurites, whereas after treatment with GCM these puncta clustered at distinct spots along neurites (Fig. 3).

Taken together, GCM and cholesterol enhanced the number of synapses and their spontaneous and evoked activity not immediately, but slowly within several days. Both induced paired-pulse depression after 1 day of treatment. Cholesterol induced a smaller increase in synapse number than GCM, but fully mimicked the GCM-induced increase in synaptic activity.

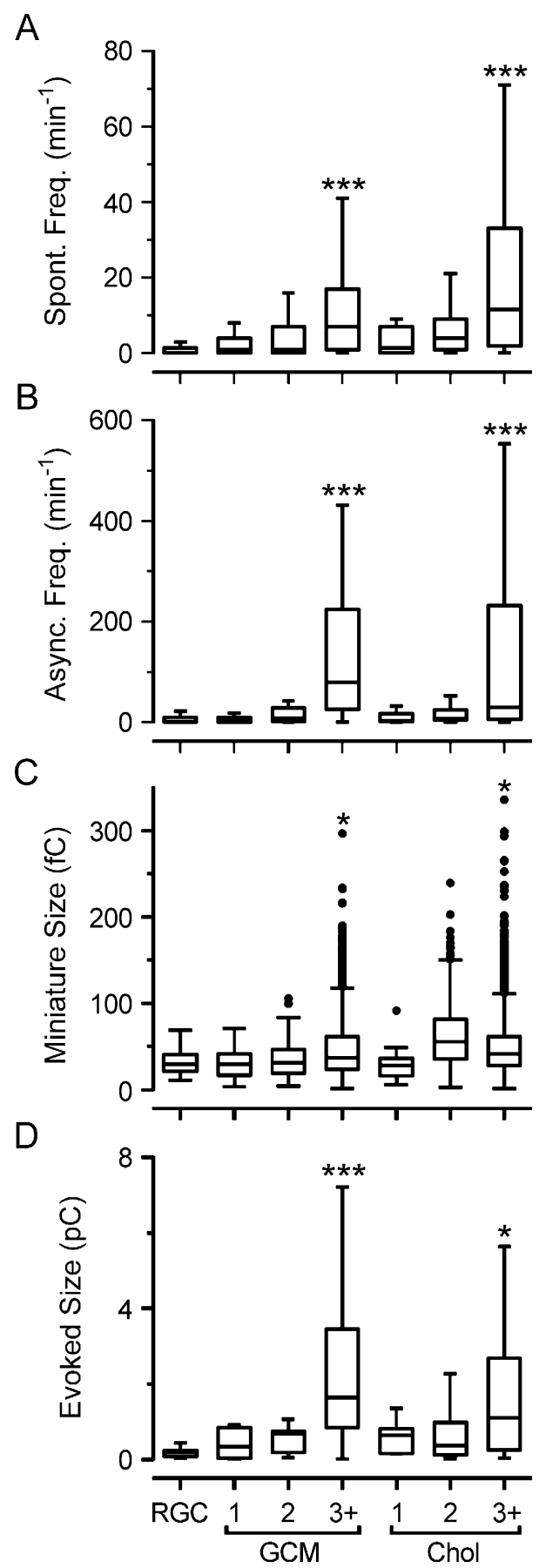



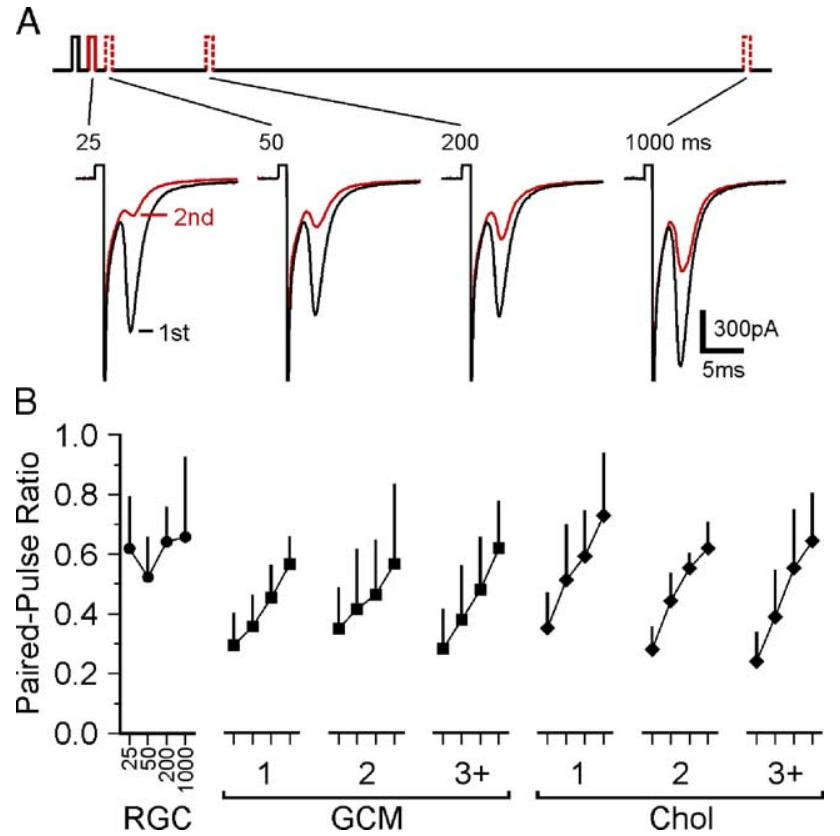

Fig. 2. GCM and cholesterol induce paired-pulse depression within 1 day of treatment. (A) Current traces showing pairs of EPSCs evoked at different ISIs (25, 50, 200 and $1000 \mathrm{~ms}$; 1st: black, 2nd: red) in an RGC cultured with GCM. Each trace represents the average of ten consecutively evoked currents. (B) PPRs (mean \pm SD) for indicated ISIs in RGCs cultured for 5 days in defined medium and then for 1,2 or at least 3 days in defined medium (RGC; circles, $n=6$ ), in GCM (GCM; squares, $1: 6 ; 2: 6 ; 3+: 35$ ) or in cholesterol (Chol; diamonds, $1: 5 ; 2: 7 ; 3+: 32$ ). GCM- and cholesterolinduced changes in the PPR at $25 \mathrm{~ms}$ ISI were statistically significant compared to untreated controls $(P<0.05$; ANOVA, Dunnett's test).

Time course of GCM- and cholesterol-induced increase in neuritic cholesterol content

The long-delay, with which GCM and cholesterol promoted synaptogenesis, contrasted with the notion that synapses form within $1 \mathrm{~h}$ (Ahmari et al., 2000; Friedman et al., 2000; Gomperts et al., 2000) and suggested the presence of a so far undefined, rate-limiting process. As a first candidate, we tested whether the neuronal uptake of cholesterol caused the delay. Using staining with filipin, a fluorescent sterol-binding antibiotic, we determined how fast GCM and cholesterol raise the level of cholesterol in RGCs. As shown in Fig. 4, GCM enhanced the cholesterol content of neurites gradually reaching a 10-fold increase within $72 \mathrm{~h}$. Directly added cholesterol had the same effect, but with a different time course: the cholesterol content increased by 20 -fold within $48 \mathrm{~h}$, but then declined to a very similar level as with GCM treatment. The increase in filipin content could have been caused by an increase in the density of neurites rather than their cholesterol content. However, this appeared unlikely, because a corresponding 20fold increase in neurite density was never observed and because the cholesterol level in cholesterol-treated cultures declined after $48 \mathrm{~h}$. To further test for this possibility, RGCs were cultured as for filipin staining but labeled with the membrane marker DiI. As shown in Fig. 4, in contrast to filipin, DiI fluorescence did not change in cells treated with GCM or cholesterol (Fig. 4) indicating that the increase in filipin fluorescence reflected an increase in cholesterol content rather than neurite density. The faster increase in cholesterol- compared to GCM-treated cultures was probably caused by the fact that directly added cholesterol is transferred directly to cells by the medium component albumin (Brown and Goldstein, 1986), whereas cholesterol contained in GCM is taken up by regulated endocytosis of lipoproteins. Despite the different rates of cholesterol- and GCM-induced increase in neuritic cholesterol content, both enhanced synapse numbers with a rather similar delay. This excluded that the increase in cholesterol content determined the rate of synaptogenesis.

Independence of cholesterol-induced effects on synaptic activity from steroid- or hedgehog-mediated signaling

Cholesterol is the obligatory precursor for steroids (Baulieu et al., 2001) and thus it appeared possible that the delayed effects on synapses were mediated by steroids, which may be synthesized by RGCs from added cholesterol and which have been shown to affect synapses (McEwen, 2002). We tested whether treatment of RGC microcultures with pregnenolone, a steroid that is derived from cholesterol and that serves as precursor for other steroids, mimicked the effects of cholesterol on synaptic activity. However, in microcultures treated for 6 days with pregnenolone $(10 \mathrm{nM}$ to $8 \mu \mathrm{M})$, the frequencies of spontaneous (1st quartile/median/3rd quartile: $0 / 0 / 1 \mathrm{~min}^{-1}$ ) and asynchronous release $\left(0 / 0 / 1.5 \mathrm{~min}^{-1} ; n=32\right)$ were similarly low as in untreated cultures (spont: 0/0/1 $\mathrm{min}^{-1}$; async: 0/0/1 $\mathrm{min}^{-1}$; $n=51$ ), whereas RGCs that were treated in parallel with cholesterol showed enhanced synaptic activity (spont: 0/2/24 $\min ^{-1}$; async: $\left.0 / 10.5 / 83 \min ^{-1} ; n=90\right)$. Notably, RGCs were chronically exposed to progesterone $(200 \mathrm{nM})$, which is contained in the culture medium.

Alternatively, the delay in cholesterol-induced effects could have been caused by hedgehog signaling. Hedgehog is a secreted protein that acts via changes in gene expression (Lum and Beachy, 2004). It has been shown that cholesterol can be attached to hedgehog by posttranslational modification (Mann and Beachy, 2004) and that RGCs produce hedgehog (Dakubo et al., 2003). To test for this possibility, we treated RGCs with GCM in the presence or absence of cyclopamine, a plant-derived alkaloid that blocks hedgehog signaling downstream of the sterol modification (Cooper et al., 1998). However, in RGCs treated for 1 week with cyclopamine $(1 \mu \mathrm{M})$ and GCM, the frequency of asynchronous release was similar $\left(0 / 9.25 / 117 \mathrm{~min}^{-1} ; n=16\right)$ as in those treated with GCM alone $\left(0 / 15 / 32.5 \mathrm{~min}^{-1} ; n=12\right.$ neurons). Taken together, these results indicate that cholesterol enhances synaptic activity not via steroid- or hedgehog-dependent signaling pathways.

Dendrite differentiation as rate-limiting step for GCM- and cholesterol-induced synaptogenesis

A critical hint to the rate-limiting step for cholesterol-induced synaptogenesis came from a closer inspection of the GluR2/3 immunostaining. We observed that GCM and cholesterol strongly increased the density of GluR2/3 in neurites, whose form reminded of dendrites (Fig. 3). This prompted us to test whether RGCs form dendrites in microcultures and whether their development causes the delay in synaptic effects. Immunostaining with an antibody against microtubule-associated protein 2 (MAP2) revealed that after 10 to 14 days under control conditions less 
A
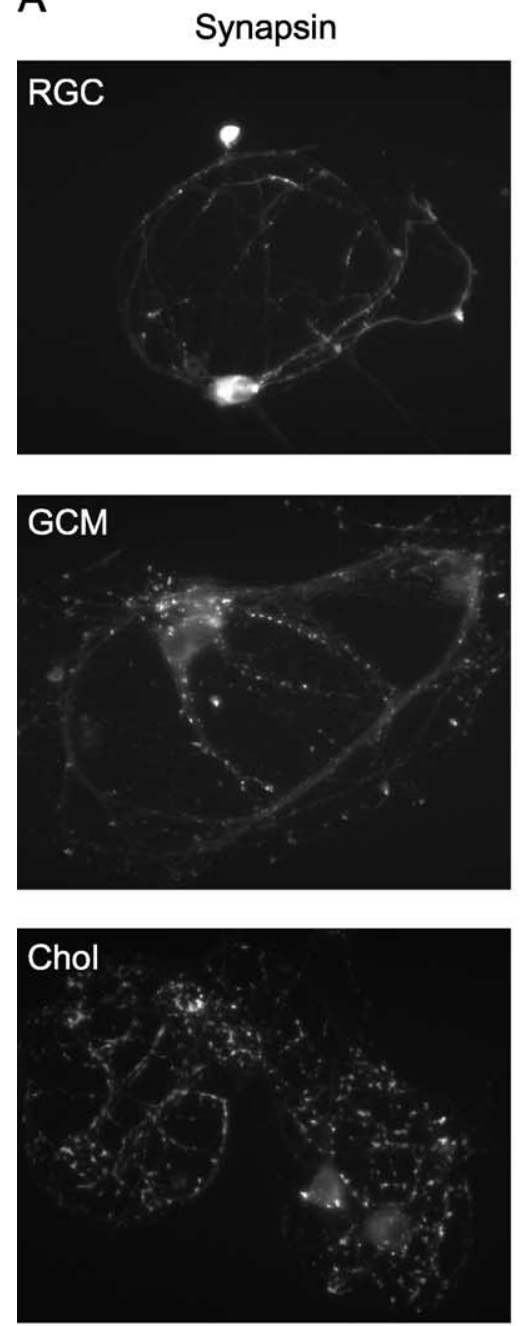

GluR2/3
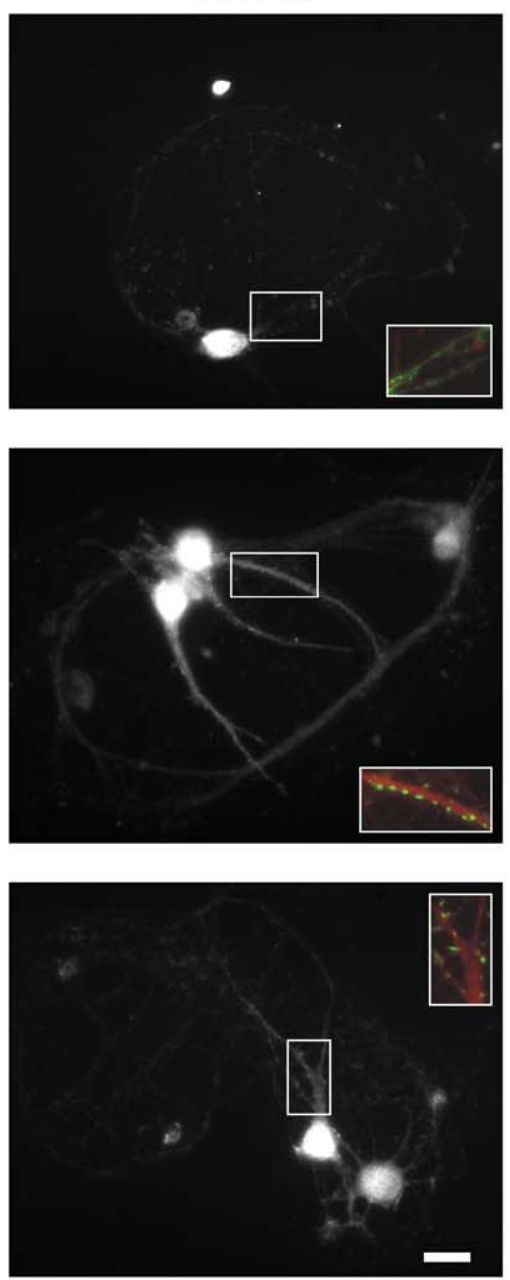

$20 \mu \mathrm{m}$
B
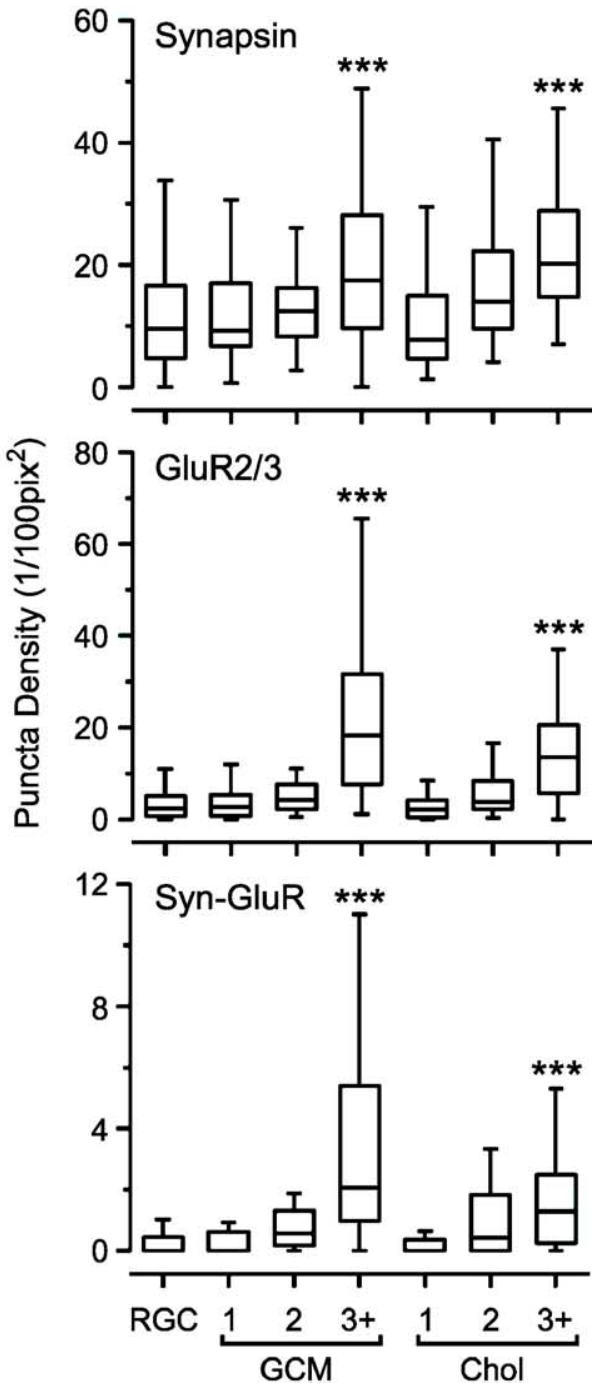

Fig. 3. GCM- and cholesterol-induced increase in synapse density requires at least 3 days of treatment. (A) Fluorescence micrographs of RGCs that were cultured for 5 days in defined medium, then for 6 days in defined medium (top), with GCM (middle) or with cholesterol (bottom) and then stained with antibodies against synapsin I (left column) and GluR2/3 (right column). Inserts show overlays of synapsin (green) and GluR2/3 (red) fluorescence in indicated areas at 1.5-fold magnification. (B) Densities of synapsin- (top), GluR2/3- (middle) and synapsin-GluR2/3-positive (bottom) puncta on neuritic processes of RGCs cultured for 5 days in defined medium and then for 1,2 or at least 3 days in defined medium (RGC; $n=99$ neurons), in GCM $(1: 31 ; 2: 21 ; 3+: 54)$ or in cholesterol (Chol; 1 : 32; 2: 21;3+: 55). Three or more days of treatment with GCM or cholesterol induced statistically significant changes compared to untreated controls (ANOVA, Dunnett's post-hoc test).

than $20 \%$ of the RGCs tested showed MAP2-positive dendrites (Fig. 5), although all RGCs showed MAP2-positive somata and formed multiple neurites (Fig. 5A, Supplementary Fig. 1). This implied that, under control conditions, RGCs grow multiple neurites but fail to differentiate MAP2-positive dendrites. The lack of dendrites can explain the absence of GluR2/3 in neurites (Fig. 3) under glia-free conditions, since GluRs are selectively targeted to mature dendrites (Craig et al., 1993). We next treated microcultures with GCM or cholesterol for different periods of time and counted the number of MAP2-positive dendrites per soma. GCM and cholesterol gradually increased the fraction of RGCs with one or more MAP2-positive dendrites reaching statistically significant changes compared to control cultures after at least 3 days of treatment (Fig. 5B). This time course was remarkably similar to the slow increase in synapse number indicating that dendrite differentiation limits the rate and extent of synaptogenesis in RGCs. GCM and cholesterol may have increased the content of MAP2 in neurites by enhancing its expression. However, immunoblots of cell extracts showed that RGCs synthesized MAP2 under glia-free condition and that GCM or cholesterol did not enhance its protein level or phosphorylation status (Fig. 6). Even in the presence of GCM or cholesterol, about $40 \%$ of neurons lacked dendrites. Previous observations that RGCs impede dendrite development in neighboring cells (Eysel et al., 1985; Perry and Linden, 1982) prompted us to determine whether a similar effect occurred in our microcultures. Indeed, we found that the percentage of RGCs lacking MAP2-positive dendrites was three- to four-fold higher in neurons growing with one or more 
A

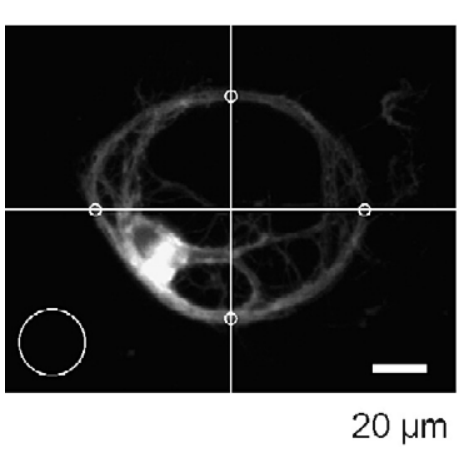

B

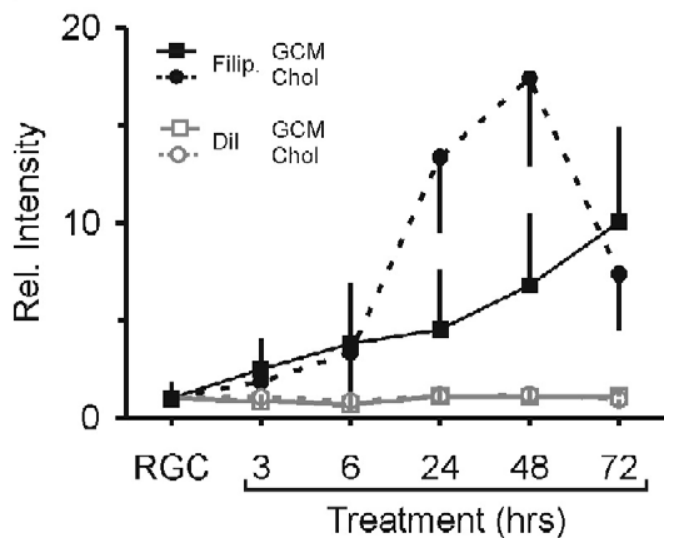

Fig. 4. Time course of GCM- and cholesterol-induced increase in neuritic cholesterol content. (A) Fluorescence micrograph of an RGC cultured for 5 days in defined medium and then for $48 \mathrm{~h}$ with GCM. Subsequently, the culture was fixed and stained with filipin. Circles indicate regions, where filipin intensity was determined in neurites (small, intersections with center lines) and in background (large). (B) Relative mean fluorescence intensities of filipin (solid black symbols, black lines) and DiI (empty gray symbols, gray lines) in neurites of RGCs cultured for 5 days in defined medium and then for the indicated times with GCM (rectangles, solid line; RGC: $n=173$ (filipin)/58 (DiI) cells; 3 h: 36/10; 6: 40/11; 24: 37/11; 48: 39/11; 72: 28/12) or with cholesterol (circles, dashed line; RGC: 110/58; 3h: 33/11; 6: 34/11; 24: 23/11; 48: 23/11; 72: 44/11). Fluorescence intensities for each time point were normalized to intensities of untreated control cells (RGC). Whiskers indicate standard deviation.
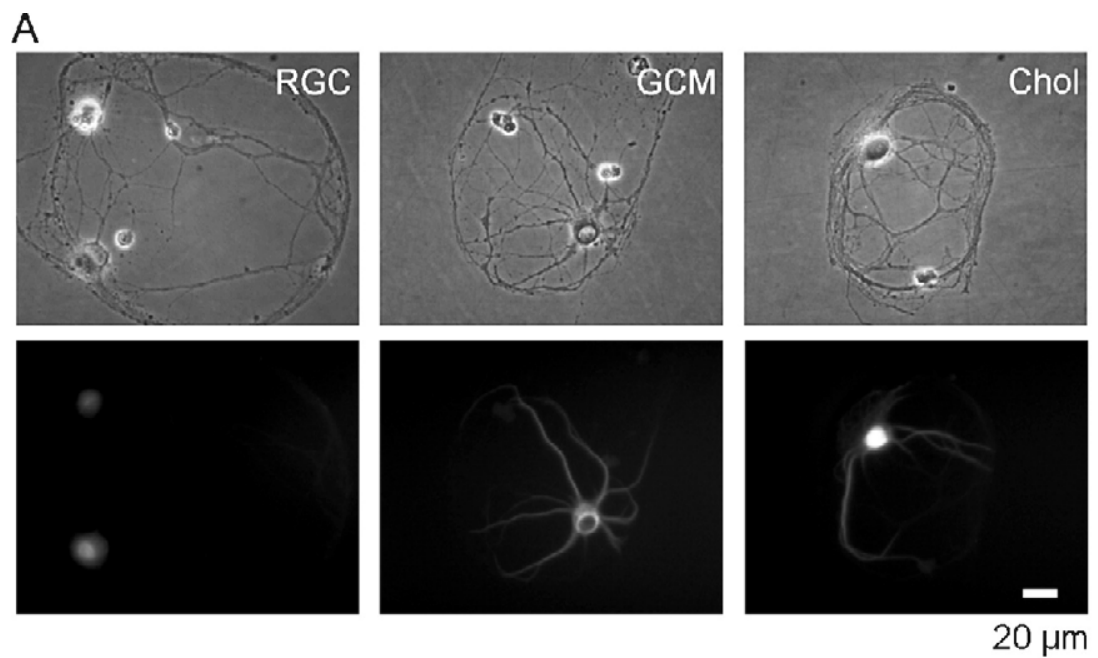

B

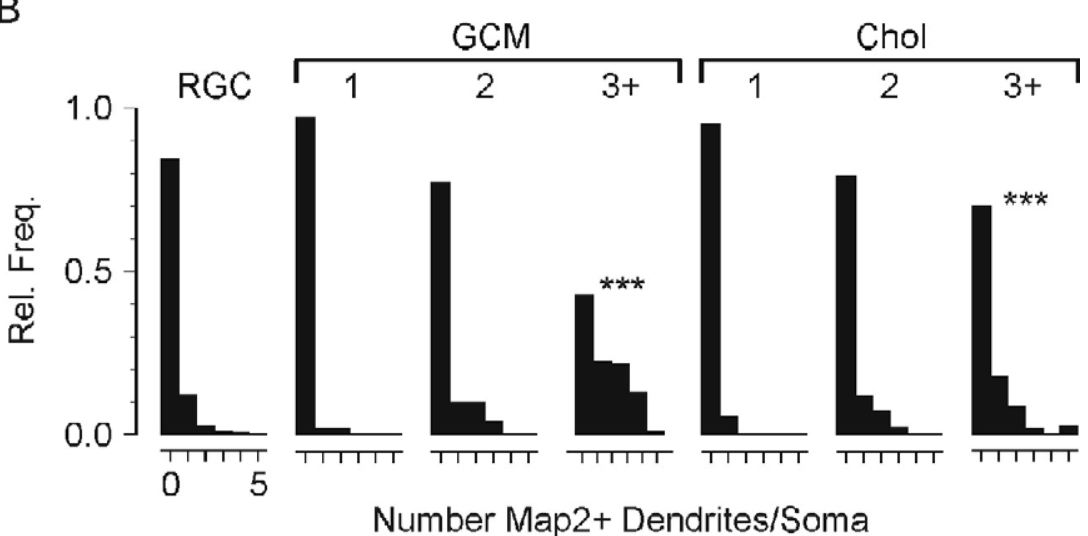

Fig. 5. Dendrite formation limits the rate of GCM- and cholesterol-induced synaptogenesis. (A) Phase-contrast (top) and fluorescence (bottom) micrographs of RGCs growing for 5 days in defined medium and then for 3 days in defined medium (left), in GCM (middle) and in cholesterol (right). Cultures were stained with antibodies against the dendritic marker MAP2. (B) Relative frequency histograms of the number of MAP2-positive dendrites per soma in RGCs cultured for 5 days in defined medium and then for 1,2 or at least 3 days in defined medium (RGC; $n=138$ neurons), in GCM $(1: 20 ; 2: 27 ; 3+: 77)$ or in cholesterol (Chol; $1: 26 ; 2: 25 ; 3+: 91)$. Only 3 to 7 days of treatment with GCM or cholesterol induced statistically significant changes compared to untreated cultures (Pearson's $\chi^{2}$ test). 


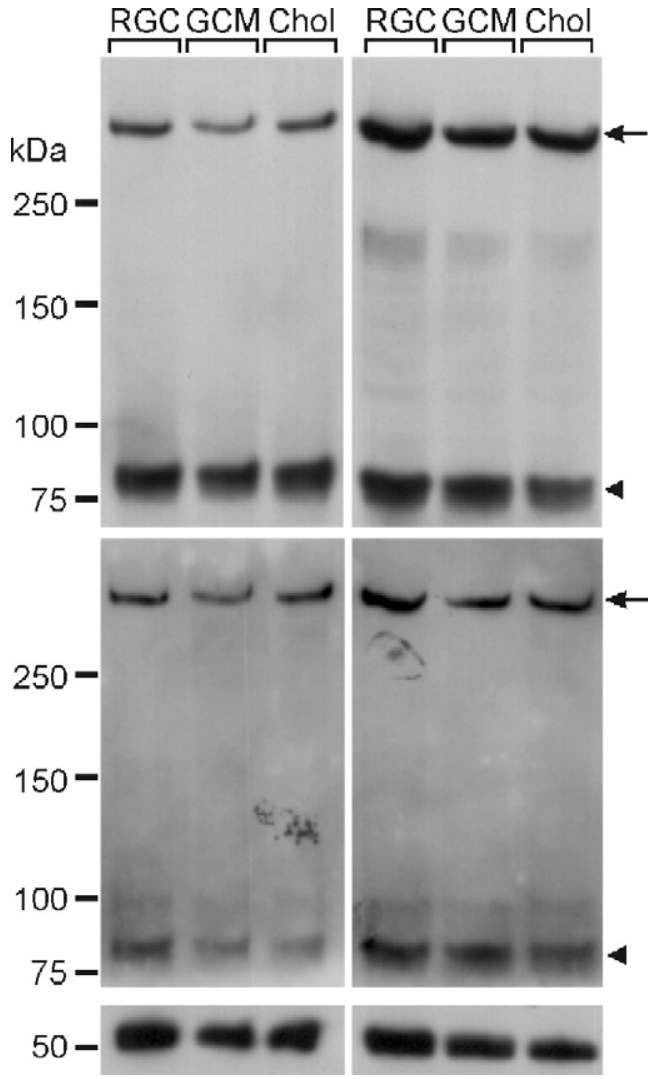

Fig. 6. GCM or cholesterol do not change MAP2 protein level and phosphorylation. Immunoblots of RGCs growing for 4 days in defined medium and then for 2 days in the presence of defined medium (RGC), GCM or cholesterol using antibodies against specific phosphorylation sites (top left, thr1620/23; top right, ser136), total MAP2 (middle panel, stripped blots from top panel) and against tubulin (bottom). Arrows and arrowheads indicate high and low molecular weight MAP2, respectively. GCM (low: $122 \pm 37 \%$; high: $115 \pm 24 ; n=4$ preparations) and cholesterol (low: $103 \pm$ $42 \%$; high: $88 \pm 16 ; n=4$ ) did not change normalized MAP2 protein levels compared to untreated controls.

neighbors (43\%) than in those growing singly on a microisland (12\%; Fig. 7) indicating an inhibitory effect of neighboring RGCs.

In summary, our results indicate that the low incidence of synapses in RGCs under glia-free conditions was due to a lack of dendrite differentiation. GCM and cholesterol promoted this process, which acts as a rate-limiting step for the increase in synapse number and activity and which involves redistribution of MAP2 from the soma to dendrites.

\section{Effects of GCM and cholesterol on subcellular distribution of GluRs}

Given the change in MAP2 distribution, we next studied the surface expression and subcellular distribution of GluRs in RGCs using antibodies against intra- and extracellular epitopes. RGCs growing under control conditions showed GluRs on the surface of somata, but not on neurites (Fig. 8A). In GCM- and cholesteroltreated cells, GluRs appeared on the surface of dendrites (Fig. $8 \mathrm{~A}$ ), but this was not due to a change in total protein level (Fig. $8 \mathrm{C})$. This indicated that GCM and cholesterol promote the differentiation of dendrites and allow thus for the redistribution of GluRs to dendrites, where a fraction is inserted in the plasma membrane. Interestingly, all neuronal somata contained an intracellular pool of GluRs regardless of the culture condition. However, its size and intrasomatic distribution varied largely from cell to cell ranging from Golgi-like patterns to somata that were entirely filled with GluRs (Figs. 3 and 8B). This variability is probably due to variable, cell-specific levels of receptor synthesis.

\section{Effects of GCM/cholesterol removal on synaptic activity}

We next tested whether the GCM-induced increase in synaptic activity persisted after removal of GCM or whether cholesterol or other glial factors were required to maintain synaptic activity. To address this, RGCs were cultured for 4 days under control conditions and then treated for 5 days with GCM to stimulate synapse formation and synaptic activity. At this point, the GCM-induced baseline synaptic activity was recorded (Fig. 9A, inset). Then, GCM was removed, cells were washed once with PBS to remove glial factors and cultured in defined medium or in the presence of GCM or of cholesterol. After further 6 days of culture (total 15 days in vitro), synaptic activity was recorded to detect its sensitivity to the removal of GCM or its replacement by cholesterol (Fig. 9A, inset). In RGC cultures, from which GCM was removed, the frequency of spontaneous and asynchronous release (Fig. 9A) and the quantal size (Fig. 9B) remained at the baseline level. In RGC cultures, in which GCM treatment was continued, the frequencies of spontaneous and asynchronous release increased further and this increase persisted if GCM was replaced by cholesterol (Fig. 9A). This indicated that RGCs continue to form synapses in vitro if provided with cholesterol. Remarkably, removal of GCM caused a significant decrease in the size of evoked EPSCs compared to the baseline level and a significant increase in the fraction of neurons, where stimulation failed to evoke EPSCs (Fig. 9C). This effect may, at least in part, explain the previously reported decrease in quantal content after removal of astrocytic feeding layers (Ullian et al., 2001). Again, these changes did not occur, when GCM was replaced by cholesterol (Fig. 9C). Taken together, these results showed that the continuous presence of

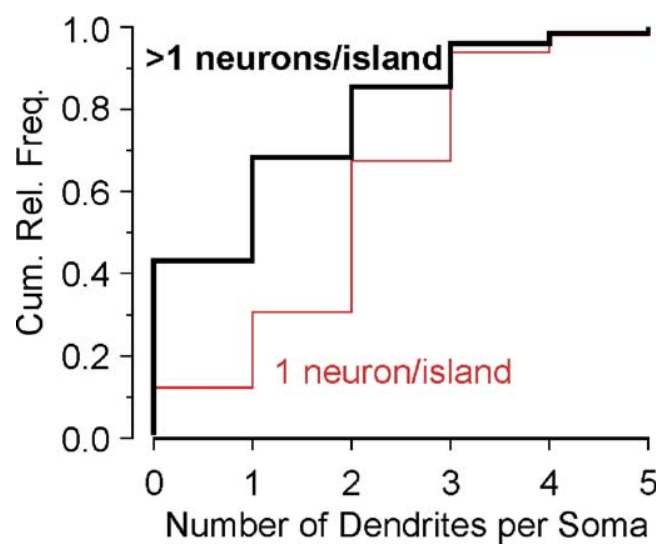

Fig. 7. Neighboring RGCs suppress dendrite differentiation in RGCs. Cumulative relative frequency plot of the number of MAP2-positive dendrites per soma for RGCs growing singly on microislands (thin red line; $n=49$ neurons) or together with at least one neuron (thick black line; $n=$ 123 neurons). The two distributions differ significantly $(P<0.001$; Pearson's $\chi^{2}$ test). Cells grew for 4 days under defined conditions and then for 4 to 6 days in the presence of GCM. 
A
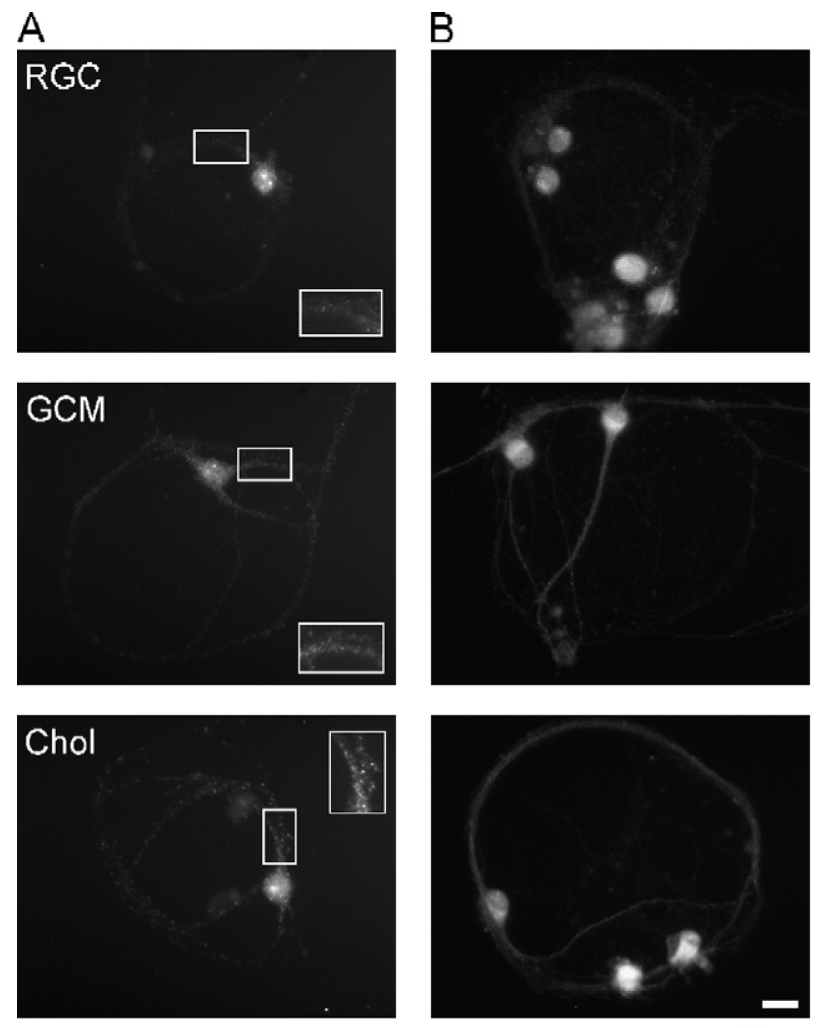

C

$20 \mu \mathrm{m}$

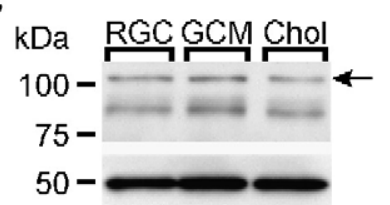

Fig. 8. GCM and cholesterol induce redistribution of GluR2/3 to dendrites. Fluorescence micrographs showing RGCs, which were grown for 5 days in defined medium and then for 5 to 8 days in the presence of defined medium (RGC, top row), GCM (middle) or cholesterol (bottom) and subsequently stained with antibodies against an extracellular (A) and intracellular (B) epitope. Inserts in panel (A) show indicated areas at 1.5-fold magnification. (C) Immunoblot of RGCs cultured as indicated reacted with antibodies against GluR2/3 (top, arrow) and tubulin (bottom). GCM (102 $\pm 43 \%$; $n=$ 3 preparations) and cholesterol (99 $\pm 40 \% ; n=3)$ did not change normalized GluR2/3 levels compared to untreated controls.

cholesterol is required to maintain evoked synaptic transmission and to support ongoing synaptogenesis.

\section{Discussion}

Our study identifies mechanisms of cholesterol-induced synaptogenesis. We show that dendrite differentiation acts as a rate-limiting step and that cholesterol is indispensable for this process. In addition, cholesterol enhances directly the differentiation of presynaptic terminals as indicated by the increase in asynchronous release and the induction of paired-pulse depression. Finally, the presence of cholesterol is essential for continuous synaptogenesis and for the functional stability of evoked transmitter release. Given that neurons survive and grow in the absence of added cholesterol, these results suggest that specific stages of neuronal differentiation depend on an external
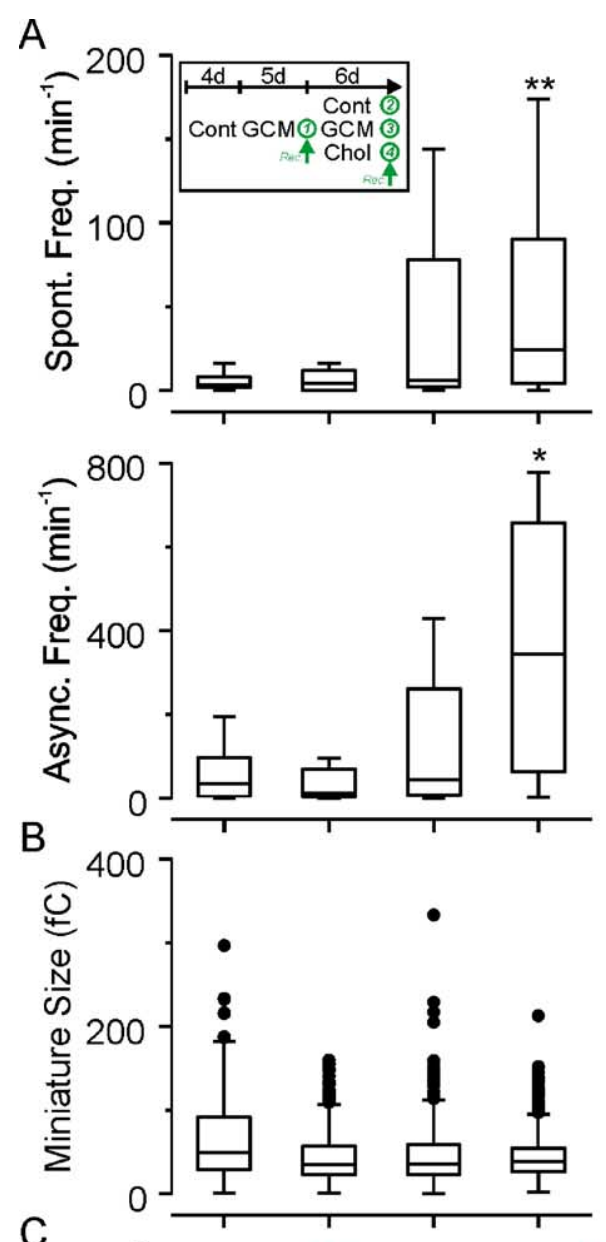

C

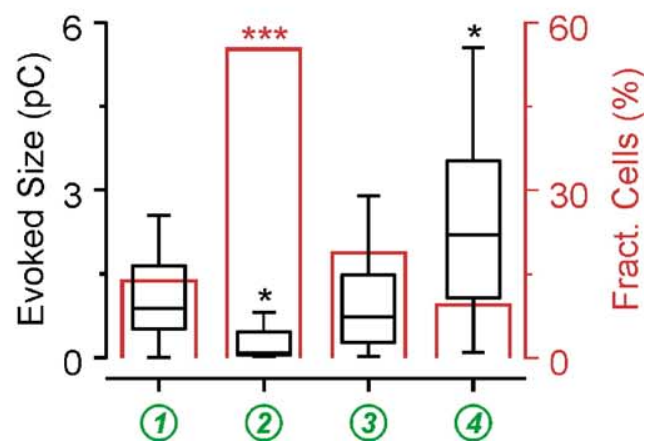

Fig. 9. Effects of GCM removal on synaptic activity. (A) Insert: Timing of culture treatment and electrophysiological recordings. Immunoisolated RGCs were cultured for 4 days in defined medium and for 5 days with GCM. Then, synaptic activity was recorded to define GCM-induced baseline activity (1). GCM was washed off with PBS and replaced by defined culture medium, GCM or medium plus cholesterol. After another 6 days in vitro, synaptic activity was recorded to define the effects of GCM removal (2), of the continued presence of GCM (3) or of its replacement by cholesterol (4) on synaptic activity. Boxplots in panel (A) show frequencies of spontaneous (upper panel) and asynchronous (lower) EPSCs. (B) Charge transfer amplitudes of miniature EPSCs. (C) Charge transfer amplitudes of evoked EPSCs (left axis, black boxplots) and fraction of neurons showing spontaneous or asynchronous events but no evoked EPSCs (right axis, red columns). After removal of GCM, the size of evoked EPSCs and the fraction of neurons lacking EPSCs decreased and increased compared to the baseline, respectively. Continued treatment with GCM or cholesterol prevented this decrease. Asterisks indicate statistically significant changes compared to baseline (A, C left axis: Kruskal-Wallis test; C right axis: Pearson's $\chi^{2}$ test). 
source of this lipid and that disturbance of cholesterol delivery by astrocytes may impair synaptic function (Pfrieger, 2003b).

\section{Dendrite differentiation limits the rate of glia-induced synaptogenesis and requires cholesterol}

Our observation that the differentiation of dendrites causes the delay in glia-induced synaptogenesis is in line with evidence that dendrite development determines the competence of neurons to form synapses (Fletcher et al., 1994) and that dendritic filopodia play an active role in the establishment of synaptic contacts (Ziv and Smith, 1996). In addition, our findings corroborate the idea that glial cells secrete dendritepromoting signals. This has been shown in vitro for RGCs isolated from embryonic chicken (Bauch et al., 1998), for sympathetic neurons from newborn mice (Tropea et al., 1988) and for cortical neurons from embryonic mice (Keith and Wilson, 2001).

So far, signals and mechanisms that shape the dendritic tree are well established (Jan and Jan, 2003; Miller and Kaplan, 2003; Whitford et al., 2002), but much less is known about the pathways that regulate the first steps of dendrite differentiation (Libersat and Duch, 2004). Our results suggest that cholesterol is essential for this process. Under control conditions, RGCs extended several neurites that branched extensively, but lacked MAP2 or GluR2/3. They produced dendritic proteins like MAP2 and GluR2/3, which due to the absence of dendrites accumulated in the soma. Cholesterol induced a redistribution of MAP2 and GluR2/3 receptors to neurites without changing their level of expression. Why does dendrite differentiation require cholesterol? Cholesterol may serve as building material, as the establishment of dendrites requires large amounts of lipids due to the dendritic presence of the endoplasmatic reticulum and the Golgi apparatus (Horton and Ehlers, 2004). A specific dependence of dendrites on the neuronal cholesterol level has been shown previously in primary cortical cultures from embryonic rats, where experimentally induced cholesterol deficiency decreased selectively the number and length of dendrites, but not axonal elongation (Fan et al., 2002). A recent study on immunoisolated RGCs growing in compartment cultures showed that the neurons require external cholesterol to regrow axons in 1 week old cultures (Hayashi et al., 2004), but the formation of dendrites was not studied.

The induction of dendrites by GCM or cholesterol may also explain the enhanced size of miniature postsynaptic currents, which was observed in about half of the RGCs tested. Large miniature currents occurred with the same delay as MAP2- and GluR2/3-positive dendrites indicating that the increasing pool of GluRs in dendrites sustained higher densities of postsynaptic GluRs and thus enhanced transmitter-sensitivity. Due to the very low incidence of dendrites under control conditions, we cannot examine a possible direct effect of cholesterol on postsynaptic receptor clustering. Such an effect has been suggested by a recent study on cultured hippocampal neurons showing that postsynaptic clusters of GluRs are sensitive to the neuronal cholesterol content (Hering et al., 2003). An alternative explanation for the increase in quantal size could be that cholesterol increased the transmitter concentration in synaptic vesicles, for example by enhancing the efficacy of glutamate transporters (Canolle et al., 2004). This appeared unlikely, however, since such an effect should have occurred along with the strong increase in neuritic cholesterol content and thus already within $24 \mathrm{~h}$ of treatment.

\section{Cholesterol directly promotes presynaptic differentiation}

Our results indicate that cholesterol directly promotes the maturation of presynaptic terminals apart from its requirement for dendrite differentiation. First, cholesterol increased the asynchronous release more strongly than the frequency of spontaneous events and the number of synapses indicating that it enlarges the release capacity of individual synapses. It may promote the synthesis and maturation of synaptic vesicles. Cholesterol appears essential for the biogenesis of secretory vesicles in neurosecretory cell lines (Thiele et al., 2000) and for the formation of complexes between vesicle proteins in vivo (Mitter et al., 2003; Pfrieger, 2003c). The idea that cholesterol is utilized for vesicle production is further supported by our observation that cholesterol enhanced the number of synapsinpositive puncta, which were dispersed along axons. These puncta probably represent clusters of surplus synaptic vesicles that cannot be delivered to synapses.

Second, cholesterol induced PPD to a similar degree as GCM. So far, an influence of soluble factors from glia on PPD has not been reported. It has been shown that coverage of synapses by processes of Bergmann glia determines the extent of PPD in the cerebellum (Araque et al., 2001; Xu-Friedman and Regehr, 2003). A study on hippocampal slices reported that depletion of cholesterol by cyclodextrine causes an increase in paired-pulse facilitation (Koudinov and Koudinova, 2001). The fact that PPD requires efficient release sites (Zucker and Regehr, 2002) indicates that cholesterol mediates the GCMinduced increase in release efficacy that we reported previously (Nagler et al., 2001). This is further supported by the fact that PPD was induced with the same delay as the previously reported drop in the failure rate of evoked responses (Nagler et al., 2001). Our observation that cholesterol induced PPD before it increased the asynchronous release and the number of synapses suggests that it enhances the efficacy of release at existing synapses independently from a general increase in their release capacity.

Cholesterol is required for continuous synapse development and the stability of evoked release

Our study shows that removal of GCM from cultured RGCs stopped the continuous increase in synaptic activity and that this did not occur if GCM was replaced by cholesterol. This indicates that RGCs require cholesterol for ongoing synapse development. After removal of GCM, synaptic activity remained at the baseline level indicating that those synapses that had formed remained active. However, removal of GCM diminished selectively the size of evoked EPSCs and increased the number of RGCs lacking evoked synaptic responses. Again, these effects did not occur when GCM was replaced by cholesterol. This result along with the observed induction of paired-pulse depression indicates that the functional stability of evoked release requires the continuous presence of cholesterol. This is further supported by previous reports that exocytosis is sensitive to the removal of cholesterol (Chamberlain et al., 2001; Lang et al., 2001; Ohara-Imaizumi et al., 2004; Pfrieger, 2003c). 


\section{Experimental methods}

\section{Microcultures of purified CNS neurons}

Microcultures were prepared as described (Nagler et al., 2001). Briefly, postnatal day 7 (P7) Wistar rats (animal facility, Faculte de Medicine, Universite Louis Pasteur, Strasbourg) were killed by decapitation according to institutional guidelines. RGCs were purified by sequential immunopanning (Barres et al., 1988) and plated at 20 cells $\mathrm{mm}^{-2}$ on tissue culture dishes $(35 \mathrm{~mm}$ Falcon, BD Biosciences, Le Pont-de-Claix, France) containing microdots of poly-D-lysine (PDL) (70-150 kDa; $100 \mu \mathrm{g} \mathrm{ml}^{-1}$ in Neurobasal; Sigma, St.-Quentin Fallavier, France) that were formed by a custom-built microatomizer. RGCs were cultured in serum-free Neurobasal medium (Meyer-Franke et al., 1995) (Invitrogen, Cergy-Pontoise, France) supplemented with B27 (Invitrogen), bovine serum albumin (BSA; $100 \mu \mathrm{g} \mathrm{ml}{ }^{-1}$; crystalline grade

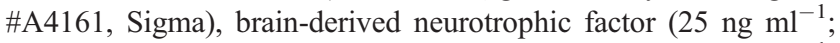
PeproTech, London, UK), ciliary neurotrophic factor $\left(10 \mathrm{ng} \mathrm{ml}^{-1}\right.$; PeproTech), forskolin (10 $\mu \mathrm{M}$; Sigma), glutamine (2 $\mathrm{mM}$; Invitrogen $)$, insulin $\left(5 \mu \mathrm{g} \mathrm{ml}{ }^{-1}\right.$; Sigma $), N$-acetylcysteine $(60 \mu \mathrm{g}$ $\mathrm{ml}^{-1}$; Sigma), penicillin (100 units $\mathrm{ml}^{-1}$; Invitrogen), progesterone (62 $\mathrm{ng} \mathrm{ml}^{-1}$; Sigma), putrescine $\left(16 \mu \mathrm{g} \mathrm{ml}^{-1}\right.$; Sigma), sodium pyruvate (1 mM; Invitrogen), sodium selenite (40 $\mathrm{ng} \mathrm{ml}^{-1}$; Sigma), streptomycin $\left(100 \mu \mathrm{g} \mathrm{ml}^{-1}\right.$; Invitrogen), transferrin $\left(100 \mu \mathrm{g} \mathrm{ml}^{-1}\right.$; Sigma) and tri-iodothyronine (40 $\mathrm{ng} \mathrm{ml}^{-1}$; Sigma). The supplemented medium is further referred to as $\mathrm{NB}+$. Three times a week, half of the culture medium was replaced by fresh $\mathrm{NB}+$. Cholesterol ( $5 \mu \mathrm{g} \mathrm{ml}^{-1}$; Sigma) was added to neuronal cultures from a $1000 \times$ ethanolic stock solution. GCM was obtained from cortical glial cells (Pfrieger and Barres, 1997). Briefly, papain-digested and triturated cortices from $\mathrm{P} 7$ rats were cultured in PDL-coated tissue culture flasks (25 $\mathrm{cm}^{2}$, TPP, Trasadingen, Switzerland) in a medium that does not support survival of neurons containing DMEM, heat-inactivated fetal calf serum (10\%), penicillin (100 units $\left.\mathrm{ml}^{-1}\right)$, streptomycin $\left(100 \mu \mathrm{g} \mathrm{ml}^{-1}\right)$, glutamine $(2 \mathrm{mM})$ and sodium pyruvate ( $1 \mathrm{mM}$, all Invitrogen). After 1 week, culture flasks were washed with PBS and glial cells were cultured in NB+ except that brain-derived neurotrophic factor, ciliary neurotrophic factor and B27 were omitted. Three times a week, half of the GCM was harvested and replaced by fresh $\mathrm{NB}+$. GCM was spun down ( 5 $\min$ at $3000 \mathrm{~g}$ ) to remove cellular debris and added to 5 day old RGC microcultures by replacing 5 out of 7 parts of culture medium with 3 parts of GCM and 2 parts of fresh NB+. To some cultures, pregnenolone (kind gift of E.E. Baulieu, Le Kremlin-Bicêtre) or cyclopamine (Sigma) were added from ethanolic or methanolic stock solutions $(1000 \times)$, respectively.

\section{Electrophysiological recordings}

Whole-cell currents were recorded at room temperature (20$24^{\circ} \mathrm{C}$; RT) on an inverted microscope (Axiovert 135TV, Zeiss, Göttingen, Germany) with patch pipettes made of borosilicate glass (2-5 M $\Omega$; PG52165-4; World Precision Instruments, Aston, UK) using an Axopatch 200B amplifier (Axon Instruments, Union City, CA, USA), a data acquisition board (PCI-MIO-16E1, National Instruments, Munich, Germany) and custom-written Labview programs (National Instruments). The intracellular recording solution contained (in $\mathrm{mM}$, all Sigma) 100 potassium gluconate, $10 \mathrm{KCl}, 10$ EGTA, 10 HEPES adjusted to $\mathrm{pH} 7.4$ with $\mathrm{KOH}$. The extracellular solution contained (in $\mathrm{mM}$, all Sigma)
$120 \mathrm{NaCl}, 3 \mathrm{CaCl}_{2}, 2 \mathrm{MgCl}_{2}, 5 \mathrm{KCl}, 10$ HEPES adjusted to $\mathrm{pH}$ 7.4 with $\mathrm{NaOH}$. The membrane potential was clamped at -70 $\mathrm{mV}$. Currents were low-pass filtered at $5 \mathrm{kHz}$ and digitized at 20 $\mathrm{kHz}$. For each cell, spontaneously occurring postsynaptic currents were recorded during $30 \mathrm{~s}$. Then, evoked synaptic responses were elicited by extracellular field stimulation [constant current pulses, 30-70 mA/1 ms (Isolator-11, Axon Instruments) delivered via two platinum electrodes (distance $\sim 4 \mathrm{~mm}$ )] at $1 \mathrm{~Hz}$ with three trains of 20 pulses each (Nagler et al., 2001). Whole-cell currents were recorded continuously during stimulation to include asynchronous transmitter release occurring at least $20 \mathrm{~ms}$ after the stimuli (Nagler et al., 2001). For simplicity, we refer to synaptic currents that occur synchronously within $20 \mathrm{~ms}$ after a stimulus as evoked currents and to those occurring afterwards as asynchronous currents. To determine the size of miniature EPSCs (quantal size), we analyzed spontaneous (autaptic) EPSCs from singly growing RGCs, as in these cells action-potential evoked transmitter release is suppressed by the voltage-clamp (Nagler et al., 2001). Synaptic currents were analyzed with custom-written Labview routines. Spontaneous and asynchronous synaptic currents were detected automatically based on size and timing criteria. The frequency of asynchronous events was corrected for the rate of spontaneous synaptic currents. Charge transfer amplitudes of detected synaptic currents were measured by integrating the baseline-corrected membrane current over $8 \mathrm{~ms}$ starting at the onset of the synaptic current. For evoked currents, the onset was determined visually. Since evoked synaptic currents often overlapped with the large sodium current that was activated by extracellular stimulation, the averaged charge transfer of transmission failures was subtracted from evoked EPSCs. Paired-pulse responses were investigated by applying ten trains of two pulses for each ISI (25, 50, 200 and $1000 \mathrm{~ms}$ ). Charge transfer amplitudes from the ten trains were averaged and the PPR was calculated.

\section{Filipin and DiI staining}

To determine the cholesterol content in neurites, cultured neurons were fixed ( $4 \%$ formaldehyde for $30 \mathrm{~min}$; RT) and incubated with filipin (10 $\mu \mathrm{g} \mathrm{ml}^{-1}$ for $2 \mathrm{~h}$; RT; Sigma), an antibiotic that selectively binds to sterols with a hydroxy group at the 3rd carbon atom (Norman et al., 1972). Filipin fluorescence was then excited by monochromatic light (356 nm, Xenon lamp; Polychrome Junior, TILL Photonics, Munich, Germany), viewed on an upright microscope (Axioskop II FS, Zeiss) by an appropriate emission filter (Filter Set 2, Zeiss) and a $40 \times$ objective (water-immersion; N.A. 0.8, Zeiss) and digitized by an air-cooled monochrome CCD camera $(1280 \times 1024$ pixels, 8-bit digitization width, PCO Computer Optics, Kehlheim, Germany). The fluorescence intensity in neurites was averaged from four neuritic areas of $3 \times 3$ pixels each, where neurites crossed the vertical and horizontal middle axes of each image. From these values, background fluorescence (mean intensity in a cell-free area) was subtracted (Fig. 4A). Our tests showed that filipin fluorescence varied considerably between independent experiments probably due to the instability of the compound. Therefore, filipin intensities in neurites of RGCs after different periods of treatment with GCM or cholesterol were normalized to average intensities of untreated control cultures that were run in parallel. To determine possible GCM- or cholesterol-induced changes in neurite density, RGCs treated with GCM or cholesterol and control cultures were fixed with formaldehyde $\left(4 \% ; 10 \mathrm{~min}\right.$; RT) and labeled with DiI $\left(1,1^{\prime}\right.$ - 
dioctadecyl-3,3,3',3' - tetramethylindocarbocyanine perchlorate; 2 $\mu \mathrm{g} / \mathrm{ml} 30 \mathrm{~min}$, RT; Molecular Probes/Invitrogen), which under these conditions labels the plasma membrane. Notably, DiI fluorescence was viewed on the same setup as filipin using an appropriate filter set and analyzed as described for filipin.

\section{Immunocytochemistry}

Cells growing on tissue culture plates were fixed $(7 \mathrm{~min}$ in methanol at $-30^{\circ} \mathrm{C}$ ), blocked $[30 \mathrm{~min}$ at room temperature; $50 \%$ goat serum in antibody buffer containing $\mathrm{NaCl} 150 \mathrm{mM}, 50 \mathrm{mM}$ Tris, BSA 1\% (\#A2153, Sigma), L-lysine $100 \mathrm{mM}$, sodium azide $0.04 \%, \mathrm{pH} 7.4]$ and incubated overnight with primary antibodies (diluted in antibody buffer). Presynaptic terminals and postsynaptic receptor clusters were stained with a mouse monoclonal antisynapsin I antibody (Cl 46.1, 1:500, Synaptic Systems, Göttingen, Germany) and a rabbit polyclonal antibody against an intracellular epitope of GluR2/3 (1:200, Upstate Biotech/Euromedex, Mundolsheim, France; $0.2 \%$ Triton $\mathrm{X}$ added during primary incubation) and visualized with Alexa 488- (Molecular Probes/Invitrogen) and Cy3conjugated secondary antibodies (1 h room temperature; Jackson ImmunoResearch Labs, Soham, UK), respectively. Surface expression of GluRs was determined by a monoclonal antibody against an extracellular epitope of GluR2 (MAB397; 1:300; Chemicon/Euromedex, Souffelweyersheim, France). For this, cells were fixed with formaldehyde $(4 \%, 10 \mathrm{~min}, \mathrm{RT})$, incubated with primary antibody without permeabilization (overnight, $4^{\circ} \mathrm{C}$ ) followed by biotinylated secondary antibody (1 h, RT; Jackson) and streptavidin-conjugated Cy3 (1 h, RT; Jackson). Dendrites and microtubuli were visualized by mouse monoclonal antibodies against MAP2 (HM-2; 1:400; Sigma) and against $\alpha$-tubulin (3A2; 1:1000; Synaptic Systems), respectively. Immunofluorescence was viewed using an $\mathrm{Hg}$ lamp (HBO100, Zeiss), appropriate filter sets (Cy3: XF33, Cy2: XF100; Omega Optical/Photomed, Seefeld, Germany) and a $40 \times$ objective (Zeiss). Image acquisition was performed as described above. Control experiments showed low background staining by secondary antibodies (data not shown). The number of MAP2-positive dendrites per soma was counted manually (length at least one soma diameter). The densities of synapsin-, GluR2/3 and doubledstained puncta on neurites were detected automatically by a modified routine (Ma et al., 1999; Nagler et al., 2001). Images were filtered (Laplacian) and segmented [threshold: median intensity of the filtered image plus antibody-specific offsets; synapsin: 150 analog-to-digital units (adu); GluR2/3: $130 \mathrm{adu}]$ and somata were excluded by manually drawn masks. Puncta were selected based on pixel area (synapsin: 4-250; GluR2/3: 2-250 pixels) and mean fluorescence intensity (from unprocessed image; threshold intensity representing $5 \%$ of the brightest pixels plus antibody-specific offsets; synapsin: $40 \mathrm{adu}$; GluR2/3: $30 \mathrm{adu}$ ). Double-stained puncta had to meet intensity criteria of both stains and show at least two pixels overlap. To determine the neuritic area in each image, phase contrast images were processed by a Prewitt filter, segmented (threshold intensity representing $5 \%$ of the brightest pixels) and eroded to remove single-pixel particles. All white pixels in the soma-excluded area were then counted. Puncta densities are stated per $100 \times 100$ pixels.

\section{Immunoblotting}

For immunoblots, RGCs were cultured at 80 cells $\mathrm{mm}^{-2}$, washed and harvested in sample buffer $(0.125 \mathrm{M}$ Tris/HCl, $\mathrm{pH} 6.8$;
2.5\% SDS; $0.025 \%$ bromphenol blue; $10 \%$ glycerol; $1 \% \beta-$ mercaptoethanol). Proteins were separated by SDS-PAGE (5\%), transferred to nitrocellulose membranes (Amersham Biosciences, Orsay, France) and reacted with specific antibodies including polyclonal anti phospho-MAP2 (Thr1620/1623 or Ser136; Cell Signaling Technology/Ozyme, Montigny Le Bretonneux, France), monoclonal anti MAP2 (clone HM-2, Sigma), polyclonal anti GluR2/3 (Upstate) and monoclonal anti tubulin (clone 3A2, Synaptic Systems). Proteins were detected by suitable secondary antibodies and the ECL system (Amersham Biosciences). To compare levels of phosphorylated versus total MAP2, blots reacted with phospho-specific antibodies were stripped (Restore WB Stripping buffer, Pierce/Perbio Science, Brebieres, France), reblocked and reacted with anti MAP2 antibody. Efficient removal of phospho-specific antibodies was confirmed by incubation with ECL reagent and film exposure after stripping. For quantitation, blots were digitized and intensities of background-corrected protein bands were determined using suitably sized masks. For each culture preparation, protein levels of GluR2/3 and MAP2 were normalized to tubulin levels of RGCs growing under respective conditions.

\section{Statistical analysis}

Statistical analysis of data was performed using STATISTICA 7 (StatSoft Inc., Tulsa, USA). Levels of significance are indicated by asterisks $(* P<0.05 ; * * P<0.01 ; * * * P<0.001)$.

\section{Note added in proof}

A recent publication (Christopherson et al., 2005) provides evidence that thrombospondin acts as a glia-derived synaptogenic factor in cultures of purified RGCs.

\section{Acknowledgments}

We thank D. Bagnard, T. Claudepierre and E. Mohier for helpful comments on earlier versions of the manuscript. This work was supported by the Ara Parseghian Medical Research Foundation, the CNRS, the Deutsche Forschungsgemeinschaft (PF 349/2-1), the Fondation Electricite de France, the Max-Planck Gesellschaft and the Region Alsace.

\section{Appendix A. Supplementary data}

Supplementary data associated with this article can be found, in the online version, at doi:10.1016/j.mcn.2005.02.006.

\section{References}

Ahmari, S.E., Buchanan, J., Smith, S.J., 2000. Assembly of presynaptic active zones from cytoplasmic transport packets. Nat. Neurosci. 3, $445-451$

Araque, A., Carmignoto, G., Haydon, P.G., 2001. Dynamic signaling between astrocytes and neurons. Annu. Rev. Physiol. 63, 795-813.

Barres, B.A., Silverstein, B.E., Corey, D.P., Chun, L.L.Y., 1988. Immunological, morphological, and electrophysiological variation among retinal ganglion cells purified by panning. Neuron 1, 791-803. 
Bauch, H., Stier, H., Schlosshauer, B., 1998. Axonal versus dendritic outgrowth is differentially affected by radial glia in discrete layers of the retina. J. Neurosci. 18, 1774-1785.

Baulieu, E.E., Robel, P., Schumacher, M., 2001. Neurosteroids: beginning of the story. Int. Rev. Neurobiol. 46, 1-32.

Brown, M.S., Goldstein, J.L., 1986. A receptor-mediated pathway for cholesterol homeostasis. Science 232, 34-47.

Campbell, K., Gotz, M., 2002. Radial glia: multi-purpose cells for vertebrate brain development. Trends Neurosci. 25, 235-238.

Canolle, B., Masmejean, F., Melon, C., Nieoullon, A., Pisano, P., Lortet, S., 2004. Glial soluble factors regulate the activity and expression of the neuronal glutamate transporter EAAC1: implication of cholesterol. J. Neurochem. 88, 1521-1532.

Chamberlain, L.H., Burgoyne, R.D., Gould, G.W., 2001. SNARE proteins are highly enriched in lipid rafts in PC12 cells: implications for the spatial control of exocytosis. Proc. Natl. Acad. Sci. U. S. A. 98, $5619-5624$.

Christopherson, K.S., Ullian, E.M., Stokes, C.C., Mullowney, C.E., Hell, J.W., Agah, A., Lawler, J., Mosher, D.F., Bornstein, P., Barres, B.A., 2005. Thrombospondins are astrocyte-secreted proteins that promote CNS synaptogenesis. Cell 120, 421-433.

Cooper, M.K., Porter, J.A., Young, K.E., Beachy, P.A., 1998. Teratogenmediated inhibition of target tissue response to Shh signaling. Science 280, 1603-1607.

Craig, A.M., Blackstone, C.D., Huganir, R.L., Banker, G., 1993. The distribution of glutamate receptors in cultured rat hippocampal neurons: postsynaptic clustering of AMPA-selective subunits. Neuron 10, 1055-1068.

Dakubo, G.D., Wang, Y.P., Mazerolle, C., Campsall, K., McMahon, A.P., Wallace, V.A., 2003. Retinal ganglion cell-derived sonic hedgehog signaling is required for optic disc and stalk neuroepithelial cell development. Development 130, 2967-2980.

Eysel, U.T., Peichl, L., Wassle, H., 1985. Dendritic plasticity in the early postnatal feline retina: quantitative characteristics and sensitive period. J. Comp. Neurol. 242, 134-145.

Fan, Q.W., Yu, W., Gong, J.S., Zou, K., Sawamura, N., Senda, T., Yanagisawa, K., Michikawa, M., 2002. Cholesterol-dependent modulation of dendrite outgrowth and microtubule stability in cultured neurons. J. Neurochem. 80, 178-190.

Fletcher, T.L., De Camilli, P., Banker, G., 1994. Synaptogenesis in hippocampal cultures: evidence indicating that axons and dendrites become competent to form synapses at different stages of neuronal development. J. Neurosci. 14, 6695-6706.

Friedman, H.V., Bresler, T., Garner, C.C., Ziv, N.E., 2000. Assembly of new individual excitatory synapses: time course and temporal order of synaptic molecule recruitment. Neuron 27, 57-69.

Goda, Y., Davis, G.W., 2003. Mechanisms of synapse assembly and disassembly. Neuron 40, 243-264.

Gomes, F.C., Spohr, T.C., Martinez, R., Moura, N., V., 2001. Cross-talk between neurons and glia: highlights on soluble factors. Braz. J. Med. Biol. Res. 34, 611-620.

Gomperts, S.N., Carroll, R., Malenka, R.C., Nicoll, R.A., 2000. Distinct roles for ionotropic and metabotropic glutamate receptors in the maturation of excitatory synapses. J. Neurosci. 20, 2229-2237.

Hama, H., Hara, C., Yamaguchi, K., Miyawaki, A., 2004. PKC signaling mediates global enhancement of excitatory synaptogenesis in neurons triggered by local contact with astrocytes. Neuron 41, 405-415.

Hayashi, H., Campenot, R.B., Vance, D.E., Vance, J.E., 2004. Glial lipoproteins stimulate axon growth of central nervous system neurons in compartmented cultures. J. Biol. Chem. 279, 14009-14015.

Hering, H., Lin, C.C., Sheng, M., 2003. Lipid rafts in the maintenance of synapses, dendritic spines, and surface AMPA receptor stability. J. Neurosci. 23, 3262-3271.

Horton, A.C., Ehlers, M.D., 2004. Secretory trafficking in neuronal dendrites. Nat. Cell Biol. 6, 585-591.

Jan, Y.N., Jan, L.Y., 2003. The control of dendrite development. Neuron 40, $229-242$
Keith, C.H., Wilson, M.T., 2001. Factors controlling axonal and dendritic arbors. Int. Rev. Cytol. 205, 77-147.

Koirala, S., Reddy, L.V., Ko, C.P., 2003. Roles of glial cells in the formation, function, and maintenance of the neuromuscular junction. J. Neurocytol. 32, 987-1002.

Koudinov, A.R., Koudinova, N.V., 2001. Essential role for cholesterol in synaptic plasticity and neuronal degeneration. FASEB J. 15, 1858-1860.

Lang, T., Bruns, D., Wenzel, D., Riedel, D., Holroyd, P., Thiele, C., Jahn, R., 2001. SNAREs are concentrated in cholesterol-dependent clusters that define docking and fusion sites for exocytosis. EMBO J. 20, $2202-2213$

Lemke, G., 2001. Glial control of neuronal development. Annu. Rev. Neurosci. 24, 87-105.

Li, Z., Sheng, M., 2003. Some assembly required: the development of neuronal synapses. Nat. Rev., Mol. Cell Biol. 4, 833-841.

Libersat, F., Duch, C., 2004. Mechanisms of dendritic maturation. Mol. Neurobiol. 29, 303-320.

Lum, L., Beachy, P.A., 2004. The Hedgehog response network: sensors, switches, and routers. Science 304, 1755-1759.

Ma, L., Zablow, L., Kandel, E.R., Siegelbaum, S.A., 1999. Cyclic AMP induces functional presynaptic boutons in hippocampal CA3-CA1 neuronal cultures. Nat. Neurosci. 2, 24-30.

Mann, R.K., Beachy, P.A., 2004. Novel lipid modifications of secreted protein signals. Annu. Rev. Biochem. 73, 891-923.

Mauch, D.H., Nägler, K., Schumacher, S., Göritz, C., Müller, E.C., Otto, A., Pfrieger, F.W., 2001. CNS synaptogenesis promoted by glia-derived cholesterol. Science 294, 1354-1357.

Mazzanti, M., Haydon, P.G., 2003. Astrocytes selectively enhance N-type calcium current in hippocampal neurons. GLIA 41, 128-136.

McEwen, B., 2002. Estrogen actions throughout the brain. Recent Prog. Horm. Res. 57, 357-384.

Meyer-Franke, A., Kaplan, M.R., Pfrieger, F.W., Barres, B.A., 1995. Characterization of the signaling interactions that promote the survival and growth of developing retinal ganglion cells in culture. Neuron 15, 805-819.

Miller, F.D., Kaplan, D.R., 2003. Signaling mechanisms underlying dendrite formation. Curr. Opin. Neurobiol. 13, 391-398.

Mitter, D., Reisinger, C., Hinz, B., Hollmann, S., Yelamanchili, S.V., Treiber-Held, S., Ohm, T.G., Herrmann, A., Ahnert-Hilger, G., 2003. The synaptophysin/synaptobrevin interaction critically depends on the cholesterol content. J. Neurochem. 84, 35-42.

Nadarajah, B., Parnavelas, J.G., 2002. Modes of neuronal migration in the developing cerebral cortex. Nat. Rev., Neurosci. 3, 423-432.

Nagler, K., Mauch, D.H., Pfrieger, F.W., 2001. Glia-derived signals induce synapse formation in neurones of the rat central nervous system. J. Physiol. 533, 665-679.

Norman, A.W., Demel, R.A., de Kruyff, B., van Deenen, L.L., 1972 Studies on the biological properties of polyene antibiotics. Evidence for the direct interaction of filipin with cholesterol. J. Biol. Chem. 247, $1918-1929$.

Ohara-Imaizumi, M., Nishiwaki, C., Kikuta, T., Kumakura, K., Nakamichi, Y., Nagamatsu, S., 2004. Site of docking and fusion of insulin secretory granules in live MIN6 beta cells analyzed by TAT-conjugated antisyntaxin 1 antibody and total internal reflection fluorescence microscopy. J. Biol. Chem. 279, 8403-8408.

Peng, H.B., Yang, J.F., Dai, Z., Lee, C.W., Hung, H.W., Feng, Z.H., Ko, C.P., 2003. Differential effects of neurotrophins and schwann cell-derived signals on neuronal survival/growth and synaptogenesis. J. Neurosci. 23, 5050-5060.

Perry, V.H., Linden, R., 1982. Evidence for dendritic competition in the developing retina. Nature 297, 683-685.

Pfrieger, F.W., 2003a. Outsourcing in the brain: do neurons depend on cholesterol delivery by astrocytes? Bioessays 25, 72-78.

Pfrieger, F.W., 2003b. Cholesterol homeostasis and function in neurons of the central nervous system. Cell. Mol. Life Sci. 60, 1158-1171.

Pfrieger, F.W., 2003c. Role of cholesterol in synapse formation and function. Biochim. Biophys. Acta 1610, 271-280. 
Pfrieger, F.W., Barres, B.A., 1997. Synaptic efficacy enhanced by glial cells in vitro. Science 277, 1684-1687.

Reddy, L.V., Koirala, S., Sugiura, Y., Herrera, A.A., Ko, C.P., 2003. Glial cells maintain synaptic structure and function and promote development of the neuromuscular junction in vivo. Neuron 40, 563-580.

Sanes, J.R., Lichtman, J.W., 1999. Development of the vertebrate neuromuscular junction. Annu. Rev. Neurosci. 22, 389-442.

Slezak, M., Pfrieger, F.W., 2003. New roles for astrocytes: regulation of CNS synaptogenesis. Trends Neurosci. 26, 531-535.

Thiele, C., Hannah, M.J., Fahrenholz, F., Huttner, W.B., 2000. Cholesterol binds to synaptophysin and is required for biogenesis of synaptic vesicles. Nat. Cell Biol. 2, 42-49.

Tropea, M., Johnson, M.I., Higgins, D., 1988. Glial cells promote dendritic development in rat sympathetic neurons in vitro. Glia 1 , $380-392$.

Ullian, E.M., Sapperstein, S.K., Christopherson, K.S., Barres, B.A., 2001. Control of synapse number by glia. Science 291, 657-661.
Ullian, E.M., Christopherson, K.S., Barres, B.A., 2004a. Role for glia in synaptogenesis. Glia 47, 209-216.

Ullian, E.M., Harris, B.T., Wu, A., Chan, J.R., Barres, B.A., $2004 b$. Schwann cells and astrocytes induce synapse formation by spinal motor neurons in culture. Mol. Cell. Neurosci. 25, 241-251.

Whitford, K.L., Dijkhuizen, P., Polleux, F., Ghosh, A., 2002. Molecular control of cortical dendrite development. Annu. Rev. Neurosci. 25, $127-149$.

Xu-Friedman, M.A., Regehr, W.G., 2003. Ultrastructural contributions to desensitization at cerebellar mossy fiber to granule cell synapses. J. Neurosci. 23, 2182-2192.

Ziv, N.E., Garner, C.C., 2004. Cellular and molecular mechanisms of presynaptic assembly. Nat. Rev., Neurosci. 5, 385-399.

Ziv, N.E., Smith, S.J., 1996. Evidence for a role of dendritic filopodia in synaptogenesis and spine formation. Neuron 17, 91-102.

Zucker, R.S., Regehr, W.G., 2002. Short-term synaptic plasticity. Annu. Rev. Physiol. 64, 355-405. 